\title{
On the Fejér means of bounded Ciesielski systems
}

\author{
by
}

Ferenc Weisz (Budapest)

Dedicated to Professor Zbigniew Ciesielski on his 65th birthday

\begin{abstract}
We investigate the bounded Ciesielski systems, which can be obtained from the spline systems of order $(m, k)$ in the same way as the Walsh system arises from the Haar system. It is shown that the maximal operator of the Fejér means of the Ciesielski-Fourier series is bounded from the Hardy space $H_{p}$ to $L_{p}$ if $1 / 2<p<\infty$ and $m \geq 0,|k| \leq m+1$. Moreover, it is of weak type $(1,1)$. As a consequence, the Fejér means of the Ciesielski-Fourier series of a function $f$ converges to $f$ a.e. if $f \in L_{1}$ as $n \rightarrow \infty$.
\end{abstract}

1. Introduction. Bounded Ciesielski systems can be obtained from the spline systems of order $(m, k)$ in the same way as the Walsh system arises from the Haar system (see Ciesielski $[2,4,6]$ ). Ciesielski proved that the maximal operator of the Fourier series with respect to these bounded Ciesielski systems is bounded on $L_{p}(1<p<\infty)$ and so the Fourier series of a function $f \in L_{p}$ converges to $f$ a.e. and in $L_{p}$ norm. Since the Ciesielski systems are uniformly bounded, due to a theorem of Bochkarev [1], this theorem does not hold for functions in $L_{1}$. Moreover, there is $f \in L_{1}$ such that the Ciesielski-Fourier series diverges a.e. (see Kazarian and Sargsian [8]).

In this paper we extend the preceding convergence result to $L_{1}$ as follows. We investigate the arithmetic or Fejér means $\sigma_{n}^{(m, k)} f$ of the CiesielskiFourier series of $f$ and verify that $\sigma_{n}^{(m, k)} f \rightarrow f$ a.e. as $n \rightarrow \infty$ provided that $f \in L_{1}$.

2000 Mathematics Subject Classification: Primary 46B15, 41A15; Secondary 42B08.

Key words and phrases: spline systems, Hardy spaces, $p$-atom, atomic decomposition, interpolation, Fejér means.

This paper was written while the author held a research positiom at Humboldt University in Berlin supported by the Alexander von Humboldt Foundation. This research was also supported by the Hungarian Scientific Research Funds (OTKA) No F019633, by the Foundation for Research and Improvement in Higher Education No FKFP-0228/1999 and by the Széchenyi Professorship. 
We also consider the Hardy spaces $H_{p}$ on the unit interval and prove that the maximal operator $\sigma_{*}^{(m, k)}$ is bounded from $H_{p}$ to $L_{p}$ for $1 / 2<p<\infty$, if $|k| \leq m+1$. It follows by interpolation that $\sigma_{*}^{(m, k)}$ is also of weak type $(1,1)$, i.e.

$$
\sup _{\varrho>0} \varrho \lambda\left(\sigma_{*}^{(m, k)} f>\varrho\right) \leq C\|f\|_{1} \quad\left(f \in L_{1}\right) .
$$

The usual density argument then implies the above convergence result.

The same results for the Fejér means of the Walsh-Fourier series are due to the author [16].

I would like to thank Professor Ciesielski for helpful discussions while I was visiting the Mathematical Institute in Sopot.

2. Hardy spaces on the unit interval. We consider the unit interval $[0,1)$ with the Lebesgue measure $\lambda$. We briefly write $L_{p}$ for the real $L_{p}([0,1), \lambda)$ space; the norm (or quasinorm) of this space is defined by $\|f\|_{p}:=\left(\int_{[0,1)}|f|^{p} d \lambda\right)^{1 / p}(0<p \leq \infty)$.

In order to have a common notation for the dyadic and classical Hardy spaces we define the Poisson kernels $P_{t}^{(m, k)}$. If $k \leq m$ then let

If $k=m+1$ then let

$$
P_{t}^{(m, k)}(x):=\frac{c t}{\left(t+|x|^{2}\right)} \quad(x \in \mathbb{R}, t>0) .
$$

$$
P_{t}^{(m, k)}(x):=1_{\left[0,2^{-n}\right)}(x) \quad \text { if } n \leq t<n+1 \quad(x \in \mathbb{R}) .
$$

For a tempered distribution $f$ the non-tangential maximal function is defined by

$$
f_{*}^{(m, k)}(x):=\sup _{t>0}\left|\left(f * P_{t}^{(m, k)}\right)(x)\right| \quad(x \in \mathbb{R})
$$

where $*$ denotes convolution.

For $0<p<\infty$ the Hardy space $H_{p}^{(m, k)}(\mathbb{R})$ consists of all tempered distributions $f$ for which

Now let

$$
\|f\|_{H_{p}^{(m, k)}(\mathbb{R})}:=\left\|f_{*}^{(m, k)}\right\|_{p}<\infty .
$$

$$
H_{p}:=H_{p}^{(m, k)}([0,1)):=\left\{f \in H_{p}^{(m, k)}(\mathbb{R}): \operatorname{supp} f \subset[0,1)\right\} .
$$

Obviously, $H_{p}$ is the dyadic Hardy space if $k=m+1$. It is known (see Stein [13]) that the space $H_{p}$ can be identified with $L_{p}$ if $1<p<\infty$.

A function $a \in L_{\infty}$ is called a $p$-atom if there exists an interval $I \subset[0,1)$ such that

(i) $\operatorname{supp} a \subset I$,

(ii) $\|a\|_{\infty} \leq|I|^{-1 / p}$,

(iii) $\int_{I} a(x) x^{j} d x=0$ where $j \in \mathbb{N}$ and $j \leq[1 / p-1]$, the integer part of $1 / p-1$. 
In the dyadic case, i.e. if $k=m+1$, we consider only dyadic intervals $I$ and instead of (iii) we assume

(iii') $\int_{I} a(x) d x=0$.

An operator $V$ which maps the set of distributions into the collection of measurable functions will be called p-quasi-local if there exists a constant $C_{p}>0$ such that

$$
\int_{[0,1) \backslash 16 I}|V a|^{p} d \lambda \leq C_{p}
$$

for every $p$-atom $a$ with support in $I$; here $16 I$ is the interval with the same center as $I$ and with length $16|I|$. The following result can be found in Weisz [16] (see also [15]):

THEOREM A. Suppose that the operator $V$ is sublinear and p-quasi-local for all $p_{0}<p \leq 1$. If $V$ is bounded from $L_{\infty}$ to $L_{\infty}$ then

$$
\|V f\|_{p} \leq C_{p}\|f\|_{H_{p}} \quad\left(f \in H_{p}\right) .
$$

Moreover, $V$ is of weak type $(1,1)$, i.e. if $f \in L_{1}$ then

$$
\sup _{\varrho>0} \varrho \lambda(|V f|>\varrho) \leq C_{1}\|f\|_{1} \text {. }
$$

3. Bounded Ciesielski systems. First we introduce the spline systems as in Ciesielski [4]. Let us denote by $D$ the differentiation operator and define the integration operators

$$
G f(t):=\int_{0}^{t} f d \lambda, \quad H f(t):=\int_{t}^{1} f d \lambda .
$$

Let $m \geq-1$ be a fixed integer and $\chi_{n}, n=1,2, \ldots$, be the Haar functions. Applying the Schmidt orthonormalization to the linearly independent functions $1, t, \ldots, t^{m+1}, G^{m+1} \chi_{n}(t), n \geq 2$, we get the spline system $\left(f_{n}^{(m)}, n \geq-m\right)$ of order $m$. For $0 \leq k \leq m+1$ and $n \geq k-m$ define the splines

$$
f_{n}^{(m, k)}:=D^{k} f_{n}^{(m)}, \quad g_{n}^{(m, k)}:=H^{k} f_{n}^{(m)}
$$

of order $(m, k)$. Let us normalize these functions and introduce a more unified notation:

$$
h_{n}^{(m, k)}:= \begin{cases}f_{n}^{(m, k)}\left\|f_{n}^{(m, k)}\right\|_{2}^{-1} & \text { for } 0 \leq k \leq m+1, \\ g_{n}^{(m,-k)}\left\|f_{n}^{(m,-k)}\right\|_{2} & \text { for } 0 \leq-k \leq m+1 .\end{cases}
$$

If $m=-1$ and $k=0$ we get the Haar system, and if $m=k=0$ the Franklin system. 
In this paper the constants $C$ and $q$ depend only on $m$ and the constants $C_{p}$ depend only on $p$ and $m$ and may be different in different contexts; $q$ always denotes a constant for which $0<q<1$.

It is proved in Ciesielski [4] that

$$
\left|h_{2^{\mu}+\nu}^{(m, k)}(t)\right| \leq C 2^{\mu / 2} q^{2^{\mu}\left|t-\nu / 2^{\mu}\right|}
$$

where $m \geq-1,|k| \leq m+1, \mu \in \mathbb{N}$ and $\nu=1, \ldots, 2^{\mu}$.

The partial sums and the maximal operator of the partial sums of the spline Fourier series are defined by

$$
P_{n}^{(m, k)} f:=\sum_{i=|k|-m}^{n}\left(f, h_{i}^{(m, k)}\right) h_{i}^{(m,-k)}
$$

and

$$
P_{*}^{(m, k)} f:=\sup _{n \in \mathbb{N}}\left|P_{n}^{(m, k)} f\right|
$$

respectively, where $m \geq-1,|k| \leq m+1$ and $(f, g)$ denotes the usual scalar product $\int_{[0,1)} f g d \lambda$.

Starting with the spline system $\left(h_{n}^{(m, k)}, n \geq|k|-m\right)$ we define the bounded Ciesielski system $\left(c_{n}^{(m, k)}, n \geq|k|-m\right)$ in the same way as the Walsh system arises from the Haar system, namely,

$$
c_{n}^{(m, k)}:=h_{n}^{(m, k)} \quad(n=|k|-m, \ldots, 1)
$$

and

$$
c_{2^{\nu}+i}^{(m, k)}:=\sum_{j=1}^{2^{\nu}} A_{i, j}^{(\nu)} h_{2^{\nu}+j}^{(m, k)} \quad\left(1 \leq i \leq 2^{\nu}\right) .
$$

Since $c_{n}^{(-1,0)}=w_{n}(n \geq 1)$ is the usual Walsh system and $h_{n}^{(-1,0)}=h_{n}$ $(n \geq 1)$ is the usual Haar system, it follows that $A_{i, j}^{(\nu)}=\left(w_{2^{\nu}+i}, h_{2^{\nu}+j}\right)$. One can show (see Ciesielski [2]) that

$$
A_{i, j}^{(\nu)}=A_{j, i}^{(\nu)}=2^{-\nu / 2} w_{i}\left(\frac{2 j-1}{2^{\nu+1}}\right) .
$$

The system $\left(c_{n}^{(m, k)}\right)$ is uniformly bounded and it is biorthogonal to $\left(c_{n}^{(m,-k)}\right)$ whenever $|k| \leq m+1$.

The partial sums, the Fejér means and the maximal operators of the Ciesielski-Fourier series are defined by

$$
C_{n}^{(m, k)} f(x):=\sum_{i=|k|-m}^{n}\left(f, c_{i}^{(m, k)}\right) c_{i}^{(m,-k)}(x)=\int_{0}^{1} D_{n}^{(m, k)}(t, x) f(t) d t,
$$




$$
\sigma_{n}^{(m, k)} f(x):=\frac{1}{n} \sum_{j=1}^{n} C_{j}^{(m, k)}(x)=\int_{0}^{1} K_{n}^{(m, k)}(t, x) f(t) d t,
$$

and

$$
C_{*}^{(m, k)} f:=\sup _{n \in \mathbb{N}}\left|C_{n}^{(m, k)} f\right|, \quad \sigma_{*}^{(m, k)} f:=\sup _{n \in \mathbb{N}}\left|\sigma_{n}^{(m, k)} f\right|,
$$

respectively, where $m \geq-1$ and $|k| \leq m+1$. Here

$$
\begin{aligned}
D_{n}^{(m, k)}(t, x) & :=\sum_{i=|k|-m}^{n} c_{i}^{(m, k)}(t) c_{i}^{(m,-k)}(x), \\
K_{n}^{(m, k)}(t, x) & :=\frac{1}{n} \sum_{j=1}^{n} D_{j}^{(m, k)}(t, x)
\end{aligned}
$$

are the Dirichlet and Fejér kernels.

Ciesielski $[5,6]$ proved that

$$
\left\|P_{*}^{(m, k)} f\right\|_{p},\left\|C_{*}^{(m, k)} f\right\|_{p} \leq C_{p}\|f\|_{p} \quad(1<p<\infty) .
$$

The Walsh-Dirichlet and Walsh-Fejér kernels $D_{n}^{(-1,0)}$ and $K_{n}^{(-1,0)}$ are denoted by $D_{n}$ and $K_{n}$, respectively. It is known (Schipp, Wade, Simon and Pál [11]) that

$$
\begin{gathered}
D_{2^{n}}(x)= \begin{cases}2^{n} & \text { if } x \in\left[0,2^{-n}\right), \\
0 & \text { if } x \in\left[2^{-n}, 1\right),\end{cases} \\
\left|K_{n}(x)\right| \leq 2 \sum_{j=0}^{N-1} 2^{j-N} \sum_{i=j}^{N-1} D_{2^{i}}\left(x+2^{-j-1}\right),
\end{gathered}
$$

where $x \in[0,1), 2^{N-1} \leq n<2^{N}$ and

$$
K_{2^{n}}(x)=C \sum_{j=0}^{n} 2^{j-n} D_{2^{n}}\left(x+2^{-j-1}\right) .
$$

Note that $\dot{+}$ denotes dyadic addition (for the definition see e.g. Schipp, Wade, Simon and Pál [11]).

4. Estimations of the Fejér kernel $K_{n}^{(m, k)}$. Write $n$ in the form $n=2^{n_{1}}+n^{(1)}$ with $2^{n_{1}}>n^{(1)}$ and denote the Rademacher functions by $r_{n}$. Set

$$
G_{\mu}^{(m, k)}(t, s):=2^{\mu / 2} r_{\mu}(s) h_{2^{\mu}+\nu}^{(m, k)}(t) \quad \text { if } \frac{\nu-1}{2^{\mu}} \leq s<\frac{\nu}{2^{\mu}}\left(1 \leq \nu \leq 2^{\mu}\right) .
$$

Then, by (2), it is easy to see that

$$
c_{2^{\mu}+\nu}^{(m, k)}(t)=\int_{0}^{1} w_{\nu}(s) r_{\mu}(s) G_{\mu}^{(m, k)}(t, s) d s=\int_{0}^{1} w_{2^{\mu}+\nu}(s) G_{\mu}^{(m, k)}(t, s) d s
$$

(see also Schipp [10] and Ciesielski, Simon and Sjölin [6]). 
THEOREM 1. We have

$$
\begin{aligned}
n K_{n}^{(m, k)}(t, x)= & D_{1}^{(m, k)}(t, x)+n^{(1)} D_{2^{n_{1}}}^{(m, k)}(t, x)+\sum_{i=0}^{n_{1}-1} 2^{i} D_{2^{i}}^{(m, k)}(t, x) \\
& +\sum_{i=0}^{n_{1}-1} L_{i}^{(m, k)}(t, x)+M_{n}^{(m, k)}(t, x)
\end{aligned}
$$

where

$$
\begin{aligned}
L_{i}^{(m, k)}(t, x) & :=\int_{0}^{1} \int_{0}^{1} r_{i}(s \dot{+} u) 2^{i} K_{2^{i}}(s+u) G_{i}^{(m, k)}(t, s) G_{i}^{(m,-k)}(x, u) d s d u, \\
M_{n}^{(m, k)}(t, x) & :=\int_{0}^{1} \int_{0}^{1} r_{n_{1}}(s \dot{+} u) n^{(1)} K_{n^{(1)}}(s+u) G_{n_{1}}^{(m, k)}(t, s) G_{n_{1}}^{(m,-k)}(x, u) d s d u .
\end{aligned}
$$

Proof. By definitions we have

(9) $n K_{n}^{(m, k)}(t, x)=2^{n_{1}} K_{2^{n_{1}}}^{(m, k)}(t, x)+\sum_{j=1}^{n^{(1)}} D_{2^{n_{1}}+j}^{(m, k)}(t, x)$

$$
=2^{n_{1}} K_{2^{n_{1}}}^{(m, k)}(t, x)+n^{(1)} D_{2^{n_{1}}}^{(m, k)}(t, x)+\sum_{j=1}^{n^{(1)}}\left(D_{2^{n_{1}}+j}^{(m, k)}(t, x)-D_{2^{n_{1}}}^{(m, k)}(t, x)\right) .
$$

By (8),

$$
\begin{aligned}
D_{2^{n_{1}}+j}^{(m, k)}(t, x) & -D_{2^{n_{1}}}^{(m, k)}(t, x)=\sum_{i=1}^{j} c_{2^{n_{1}}+i}^{(m, k)}(t) c_{2^{n_{1}}+i}^{(m,-k)}(x) \\
& =\sum_{i=1}^{j} \int_{0}^{1} \int_{0}^{1} r_{n_{1}}(s) r_{n_{1}}(u) w_{i}(s) w_{i}(u) G_{n_{1}}^{(m, k)}(t, s) G_{n_{1}}^{(m,-k)}(x, u) d s d u
\end{aligned}
$$

and so

$$
\begin{aligned}
& \sum_{j=1}^{n^{(1)}}\left(D_{2^{n_{1}}+j}^{(m, k)}(t, x)-D_{2^{n_{1}}}^{(m, k)}(t, x)\right) \\
& \quad=\int_{0}^{1} \int_{0}^{1} r_{n_{1}}(s+u) n^{(1)} K_{n^{(1)}}(s+u) G_{n_{1}}^{(m, k)}(t, s) G_{n_{1}}^{(m,-k)}(x, u) d s d u .
\end{aligned}
$$

Similarly to (9) and (10),

$$
\begin{aligned}
& 2^{n_{1}} K_{2^{n_{1}}}^{(m, k)}(t, x)=2^{n_{1}-1} K_{2^{n_{1}-1}}^{(m, k)}(t, x)+2^{n_{1}-1} D_{2^{n_{1}-1}}^{(m, k)}(t, x) \\
& \quad+\int_{0}^{1} r_{0}^{1} r_{n_{1}-1}(s+u) 2^{n_{1}-1} K_{2^{n_{1}-1}}(s+u) G_{n_{1}-1}^{(m, k)}(t, s) G_{n_{1}-1}^{(m,-k)}(x, u) d s d u .
\end{aligned}
$$


Iterating this equality, we get

$$
\begin{aligned}
& 2^{n_{1}} K_{2^{n}}^{(m, k)}(t, x)=K_{1}^{(m, k)}(t, x)+\sum_{i=0}^{n_{1}-1} 2^{i} D_{2^{i}}^{(m, k)}(t, x) \\
& +\sum_{i=0}^{n_{1}-1} \int_{0}^{1} \int_{0}^{1} r_{i}(s+\dot{+} u) 2^{i} K_{2^{i}}(s+u) G_{i}^{(m, k)}(t, s) G_{i}^{(m,-k)}(x, u) d s d u .
\end{aligned}
$$

The theorem follows from (9) and (10) and from the fact that $K_{1}^{(m, k)}(t, x)=$ $D_{1}^{(m, k)}(t, x)$.

5. The boundedness of the maximal Fejér operator on $H_{p}$. Recently the author [14] extended (3) and verified that

$$
\left\|P_{*}^{(m, k)} f\right\|_{p} \leq C_{p}\|f\|_{H_{p}} \quad\left(f \in H_{p}\right)
$$

provided that $m \geq-1,-(m+1) \leq k \leq m$ and $1 /(m-k+2)<p<\infty$. If $k=m+1$ then (11) holds for all $0<p<\infty$. It is known (see Weisz [16]) that the Walsh-Fejér means satisfy

$$
\left\|\sigma_{*}^{(-1,0)} f\right\|_{p} \leq C_{p}\|f\|_{H_{p}} \quad\left(f \in H_{p}\right)
$$

for $1 / 2<p<\infty$.

In this section we extend this inequality to bounded Ciesielski systems. To this end we need two lemmas.

Lemma 1. Suppose that $m \geq 0,|k| \leq m+1$ and $1 / 2<p<1$. If $2^{-K-1}<|I| \leq 2^{-K}$ for some $K \in \mathbb{N}$ then

$$
\begin{array}{r}
\int_{(16 I)^{\mathrm{c}}} \sup _{n \geq 2^{K}}\left(\int_{I} \frac{1}{n} \sum_{i=0}^{n_{1}-1}\left|L_{i}^{(m, k)}(t, x)\right| d t\right)^{p} d x \leq C_{p}|I|, \\
\quad \int_{(16 I)^{\mathrm{c}}} \sup _{n \geq 2^{K}}\left(\int_{I} \frac{1}{n}\left|M_{n}^{(m, k)}(t, x)\right| d t\right)^{p} d x \leq C_{p}|I| .
\end{array}
$$

If $k \leq m$ then

$$
\begin{array}{r}
\int_{(16 I)^{\mathrm{c}}} \sup _{n<2^{K}}\left(\int_{I} \frac{1}{n} \sum_{i=0}^{n_{1}-1}\left|D_{t} L_{i}^{(m, k)}(t, x)\right| d t\right)^{p} d x \leq C_{p}|I|^{1-p}, \\
\int_{(16 I)^{\mathrm{c}}} \sup _{n<2^{K}}\left(\int_{I} \frac{1}{n}\left|D_{t} M_{n}^{(m, k)}(t, x)\right| d t\right)^{p} d x \leq C_{p}|I|^{1-p},
\end{array}
$$

where $D_{t}$ denotes the $t$-derivative. 
Proof. By (6), (7) and (1) we conclude

$$
\begin{aligned}
& \left|D_{t}^{N} L_{i}^{(m, k)}(t, x)\right| \\
& \leq C 2^{i} \sum_{j=0}^{i} 2^{j} \sum_{\nu=1}^{2^{i}} \sum_{\eta=1}^{2^{i}} \int_{(\nu-1) 2^{-i}}^{\nu 2^{-i}} \int_{(\eta-1) 2^{-i}}^{\eta 2^{-i}} D_{2^{i}}\left(s \dot{+} u \dot{+} 2^{-j-1}\right) \\
& \times C 2^{i(N+2)} \sum_{j=0}^{i} 2^{j} \sum_{\nu=1}^{2^{i}} \sum_{\eta=1}^{2^{i}} \int_{(\nu-1) 2^{-i}}^{\nu 2^{-i}} \int_{(\eta-1) 2^{-i}}^{\eta(m, k)}(t)|\cdot| h_{2^{i}+\eta}^{(m,-k)}(x) \mid d s d u \\
& \times q^{2^{i} \mid t-\nu / 2^{i}} \mid q^{2^{i}\left|x-\eta / 2^{i}\right|} d s d u,
\end{aligned}
$$

where $N=0,1$. It is easy to see by (4) that $D_{2^{i}}\left(s \dot{+} u \dot{+} 2^{-j-1}\right)=0$ if $|\nu-\eta| \neq\left[2^{i-j-1}\right]$, and $D_{2^{i}}\left(s \dot{+} u \dot{+} 2^{-j-1}\right)=2^{i}$ if $|\nu-\eta|=\left[2^{i-j-1}\right]$. We can suppose that $s<u$. Hence

$$
\left|D_{t}^{N} L_{i}^{(m, k)}(t, x)\right| \leq C 2^{i(N+1)} \sum_{j=0}^{i} 2^{j} \sum_{\nu=1}^{2^{i}} q^{2^{i}\left|t-\nu / 2^{i}\right|} q^{2^{i}\left|x-\left(\nu+2^{i-j-1}\right) / 2^{i}\right|} .
$$

By the inequality

$$
\sum_{k=1}^{\infty} q^{|i-k|+|j-k|} \leq C(r) r^{|i-j|} \quad(q<r<1)
$$

(see Ciesielski, Simon and Sjölin [6]), we obtain

$$
\frac{1}{n} \sum_{i=0}^{n_{1}-1}\left|D_{t}^{N} L_{i}^{(m, k)}(t, x)\right| \leq C 2^{-n_{1}} \sum_{i=0}^{n_{1}-1} 2^{i(N+1)} \sum_{j=0}^{i} 2^{j} q^{2^{i}\left|x-t-2^{-j-1}\right|} .
$$

Assume that $n \geq 2^{K}$ and $N=0$. The last sum can be split into the sum of

$$
A_{n}(t, x):=C 2^{-n_{1}} \sum_{i=0}^{K-1} 2^{i} \sum_{j=0}^{i} 2^{j} q^{2^{i}\left|x-t-2^{-j-1}\right|}
$$

and

$$
B_{n}(t, x):=C 2^{-n_{1}} \sum_{i=K}^{n_{1}-1} 2^{i} \sum_{j=0}^{i} 2^{j} q^{2^{i}\left|x-t-2^{-j-1}\right|} .
$$

For the first sum we have

$$
A_{n}(t, x) \leq C 2^{-K} \sum_{i=0}^{K-1} 2^{i} \sum_{j=0}^{i} 2^{j} q^{2^{i}\left|x-t-2^{-j-1}\right|}
$$




$$
\begin{aligned}
= & C 2^{-K} \sum_{i=0}^{K-1} 2^{i} \sum_{j=0}^{i} 2^{j} 1_{\left\{2^{-j-1}+8 \cdot 2^{K-i} I\right\}}(x) q^{2^{i}\left|x-t-2^{-j-1}\right|} \\
& +C 2^{-K} \sum_{i=0}^{K-1} 2^{i} \sum_{j=0}^{i} 2^{j} 1_{\left\{2^{-j-1}+8 \cdot 2^{K-i} I\right\}^{\mathrm{c}}}(x) q^{2^{i}\left|x-t-2^{-j-1}\right|} \\
=: & A_{1, n}(t, x)+A_{2, n}(t, x) .
\end{aligned}
$$

Obviously,

$$
A_{1, n}(t, x) \leq C 2^{-K} \sum_{i=0}^{K-1} 2^{i} \sum_{j=0}^{i} 2^{j} 1_{\left\{2^{-j-1}+8 \cdot 2^{K-i} I\right\}}(x) .
$$

Hence

$$
\begin{aligned}
& \int_{(16 I)^{\mathrm{c}}} \sup _{n \geq 2^{K}}\left(\int_{I} A_{1, n}(t, x) d t\right)^{p} d x \\
& \leq C_{p} 2^{-K p} \int_{(16 I)^{\mathrm{c}}} \sum_{i=0}^{K-1} 2^{i p} \sum_{j=0}^{i} 2^{j p} 2^{-K p} 1_{\left\{2^{-j-1}+8 \cdot 2^{K-i} I\right\}}(x) \\
& \leq C_{p} 2^{-2 K p} \sum_{i=0}^{K-1} 2^{i p} \sum_{j=0}^{i} 2^{j p} 2^{-i} \leq C_{p}|I|
\end{aligned}
$$

On the other hand, it is easy to see that

$$
A_{2, n}(t, x) \leq C 2^{-K} \sum_{i=0}^{K-1} 2^{i} \sum_{j=0}^{i} 2^{j} q^{C 2^{i}\left|x-t_{0}-2^{-j-1}\right|}
$$

where $t_{0}$ is the center of $I$ and $t \in I$. Therefore

$$
\int_{I} A_{2, n}(t, x) d t \leq C|I|^{2} \sum_{i=0}^{\infty} 2^{i} \sum_{j=0}^{i} 2^{j} q^{C 2^{i}\left|x-t_{0}-2^{-j-1}\right|} d t .
$$

Assume that $x \notin 16 I$ and $x>t_{0}$. If $x-t_{0} \in\left[2^{-k}, 2^{-k+1}\right)$ for some $1 \leq k \leq K$, then

$$
\begin{aligned}
C|I|^{2} \sum_{i=0}^{\infty} 2^{i} \sum_{j=k}^{i} 2^{j} q^{C 2^{i}\left|x-t_{0}-2^{-j-1}\right|} & \leq C|I|^{2} \sum_{i=0}^{\infty} 2^{2 i} q^{C 2^{i}\left|x-t_{0}\right|} \\
& \leq C|I|^{2}\left|x-t_{0}\right|^{-2}
\end{aligned}
$$

In the last step we have used the inequality

$$
\sum_{\mu=0}^{\infty} 2^{\mu j} q^{2^{\mu}|t-s|} \leq C_{j}|t-s|^{-j} \quad(j>0)
$$

which can be easily seen. 
On the other hand, (21) implies

$$
C|I|^{2} \sum_{i=0}^{\infty} 2^{i} \sum_{j=0}^{(k-1) \wedge i} 2^{j} q^{2^{i}\left|x-t_{0}-2^{-j-1}\right|} \leq C|I|^{2} \sum_{j=0}^{k-1} 2^{j}\left|x-t_{0}-2^{-j-1}\right|^{-1} .
$$

Since $1 / 2<p<1$, we obtain

$$
\begin{aligned}
& \int_{(16 I)^{\mathrm{c}}} \sup _{n \geq 2^{K}}\left(\int_{I} A_{2, n}(t, x) d t\right)^{p} d x \\
& \leq C_{p}|I|^{2 p} \int_{(16 I)^{\mathrm{c}}}\left|x-t_{0}\right|^{-2 p} d x \\
& \quad+C_{p}|I|^{2 p} \sum_{k=1}^{K} \sum_{j=0}^{k-1} 2^{j p} \int_{\left\{x-t_{0} \in\left[2^{-k}, 2^{-k+1}\right)\right\}}\left|x-t_{0}-2^{-j-1}\right|^{-p} d x \\
& \leq C_{p}|I|+C_{p}|I|^{2 p} \sum_{k=1}^{K} \sum_{j=0}^{k-1} 2^{j p} 2^{-j(1-p)} \leq C_{p}|I| .
\end{aligned}
$$

The expression $B_{n}(t, x)$ can be split into the sum of

$$
B_{1, n}(t, x):=C 2^{-n_{1}} \sum_{i=K}^{n_{1}-1} 2^{i} \sum_{j=0}^{i} 2^{j} 1_{\left\{2^{-j-1}+8 I\right\}}(x) q^{2^{i}\left|x-t-2^{-j-1}\right|}
$$

and

$$
B_{2, n}(t, x):=C 2^{-n_{1}} \sum_{i=K}^{n_{1}-1} 2^{i} \sum_{j=0}^{i} 2^{j} 1_{\left\{2^{-j-1}+8 I\right\}^{\mathrm{c}}}(x) q^{2^{i}\left|x-t-2^{-j-1}\right|} .
$$

One can easily show that $1_{\left\{2^{-j-1}+8 I\right\}}(x)=0$ if $x \notin 16 I$ and $j \geq K$. Hence

$$
B_{1, n}(t, x) \leq C \sum_{j=0}^{K-1} 2^{j} 1_{\left\{2^{-j-1}+8 I\right\}}(x)
$$

and

$$
\int_{(16 I)^{\mathrm{c}}} \sup _{n \geq 2^{K}}\left(\int_{I} B_{1, n}(t, x) d t\right)^{p} d x \leq C_{p} \sum_{j=0}^{K-1} 2^{j p} 2^{-K p} 2^{-K} \leq C_{p}|I| .
$$

Moreover,

$$
B_{2, n}(t, x) \leq C 2^{-K} \sum_{i=K}^{\infty} 2^{i} \sum_{j=0}^{i} 2^{j} q^{C 2^{i}\left|x-t_{0}-2^{-j-1}\right|}
$$


and the inequality

$$
\int_{(16 I)^{\mathrm{c}}} \sup _{n \geq 2^{K}}\left(\int_{I} B_{2, n}(t, x) d t\right)^{p} d x \leq C_{p}|I|
$$

can be proved as above (cf. (20)). This together with (19), (22) and (23) implies (13).

If $n<2^{K}$ and $N=1$ then let us estimate the right hand side of (18) by

$$
C_{n}(t, x):=C \sum_{i=0}^{K-1} 2^{i} \sum_{j=0}^{i} 2^{j} q^{2^{i}\left|x-t-2^{-j-1}\right|} .
$$

The inequality

$$
\int_{(16 I)^{\mathrm{c}}} \sup _{n<2^{K}}\left(\int_{I} C_{n}(t, x) d t\right)^{p} d x \leq C_{p}|I|^{1-p}
$$

can be derived as above (see the definition of $A_{n}(t, x)$ ), which shows (15).

To prove (14) and (15) we have, by (5),

$$
\begin{aligned}
\left|D_{t}^{N} M_{n}^{(m, k)}(t, x)\right| \leq & C 2^{n_{1}(N+2)} \sum_{i=0}^{n_{1}-1} \sum_{j=0}^{i} 2^{j} \sum_{\nu=1}^{2^{n_{1}}} \sum_{\eta=1}^{2^{n_{1}}} \int_{(\nu-1) 2^{-n_{1}}}^{\nu 2^{-n_{1}}} \int_{(\eta-1) 2^{-n_{1}}}^{\eta 2^{-n_{1}}} \\
& \times D_{2^{i}}\left(s+u \dot{+} 2^{-j-1}\right) q^{2^{n_{1}}\left|t-\nu / 2^{n_{1}}\right|} q^{2^{n_{1}}\left|x-\eta / 2^{n_{1}}\right|} d s d u
\end{aligned}
$$

$(N=0,1)$. Suppose again that $\nu<\eta$. It is easy to see that for each $\nu$ there exists a set $S_{i, \nu}$ such that $D_{2^{i}}\left(s+u \dot{+} 2^{-j-1}\right)=2^{i}$ if $\eta \in S_{i, \nu}$ and $D_{2^{i}}\left(s \dot{+} u \dot{+} 2^{-j-1}\right)=0$ if $\eta \notin S_{i, \nu}$. Moreover, $\left|S_{i, \nu}\right|=2^{n_{1}-i}$ and $S_{i, \nu} \subset$ $\left[\nu+2^{n_{1}-j-1}-2^{n_{1}-i}+1, \nu+2^{n_{1}-j-1}+2^{n_{1}-i}-1\right]$. This and (17) imply

$$
\begin{aligned}
& \leq C 2^{n_{1}(N-1)} \sum_{i=0}^{n_{1}-1} 2^{i} \sum_{j=0}^{i} 2^{j} \sum_{\nu=1}^{2^{n_{1}}} \sum_{\eta-\nu=2^{n_{1}-j-1}-2^{n_{1}-i}+1}^{2^{n_{1}-j-1}+2^{n_{1}-i}-1} q^{2^{n_{1}}\left|t-\nu / 2^{n_{1}}\right|} q^{2^{n_{1}}\left|x-\eta / 2^{n_{1}}\right|} \\
& \leq C 2^{n_{1}(N-1)} \sum_{i=0}^{n_{1}-1} 2^{i} \sum_{j=0}^{i} 2^{j} \sum_{l=-2^{n_{1}-i}+1}^{2^{n_{1}-i}-1} q^{2^{n_{1}}\left|x-t-2^{-j-1}-l / 2^{n_{1}}\right|} .
\end{aligned}
$$

For (14) suppose that $n \geq 2^{K}$ and $N=0$. The last term of (24) can be split into the sum of

$$
D_{n}(t, x):=C 2^{-n_{1}} \sum_{i=0}^{K-1} 2^{i} \sum_{j=0}^{i} 2^{j} \sum_{l=-2^{n_{1}-i}+1}^{2^{n_{1}-i}-1} q^{2^{n_{1}}}\left|x-t-2^{-j-1}-l / 2^{n_{1}}\right|
$$


and

$$
E_{n}(t, x):=C 2^{-n_{1}} \sum_{i=K}^{n_{1}-1} 2^{i} \sum_{j=0}^{i} 2^{j} \sum_{l=-2^{n_{1}-i}+1}^{2^{n_{1}-i}-1} q^{2^{n_{1}}\left|x-t-2^{-j-1}-l / 2^{n_{1}}\right|} .
$$

With

$$
\begin{aligned}
D_{1, n}(t, x):= & C 2^{-K} \sum_{i=0}^{K-1} 2^{i} \sum_{j=0}^{i} 2^{j} \\
& \times \sum_{l=-2^{n_{1}-i}+1}^{2^{n_{1}-i}-1} 1_{\left\{2^{-j-1}+8 \cdot 2^{K-i} I\right\}}(x) q^{2^{n_{1}}\left|x-t-2^{-j-1}-l / 2^{n_{1}}\right|} \\
D_{2, n}(t, x):= & C 2^{-K} \sum_{i=0}^{K-1} 2^{i} \sum_{j=0}^{i} 2^{j} \\
& \times \sum_{l=-2^{n_{1}-i}+1}^{2^{n_{1}-i}-1} 1_{\left\{2^{-j-1}+8 \cdot 2^{K-i} I\right\}}(x) q^{2^{n_{1}}\left|x-t-2^{-j-1}-l / 2^{n_{1}}\right|}
\end{aligned}
$$

we have

$$
D_{n}(t, x) \leq D_{1, n}(t, x)+D_{2, n}(t, x)
$$

Then

$$
D_{1, n}(t, x) \leq C 2^{-K} \sum_{i=0}^{K-1} 2^{i} \sum_{j=0}^{i} 2^{j} 1_{\left\{2^{-j-1}+8 \cdot 2^{K-i} I\right\}}(x)
$$

and so

$$
\begin{aligned}
\int_{(16 I)^{\mathrm{c}}} \sup _{n \geq 2^{K}}\left(\int_{I} D_{1, n}(t, x) d t\right)^{p} d x & \leq C_{p} 2^{-2 K p} \sum_{i=0}^{K-1} 2^{i p} \sum_{j=0}^{i} 2^{j p} 2^{-i} \\
& \leq C_{p}|I|
\end{aligned}
$$

By an easy calculation we conclude that

$$
\begin{aligned}
D_{2, n}(t, x) \leq & C 2^{-K} \sum_{i=0}^{K-1} 2^{i} \sum_{j=0}^{i} 2^{j} \\
& \times \sum_{l=-2^{n_{1}-i}+1}^{2^{n_{1}-i}-1} 1_{\left\{2^{-j-1}+8 \cdot 2^{K-i} I\right\}^{\mathrm{c}}}(x) q^{C 2^{n_{1}}\left|x-t_{0}-2^{-j-1}\right|} \\
\leq & C 2^{n_{1}-K} \sum_{i=0}^{n_{1}-1} \sum_{j=0}^{i} 2^{j} q^{C 2^{n_{1}}\left|x-t_{0}-2^{-j-1}\right|}
\end{aligned}
$$

and 


$$
\int_{I} D_{2, n}(t, x) d t \leq C|I|^{2} 2^{n_{1}} \sum_{i=0}^{n_{1}-1} \sum_{j=0}^{i} 2^{j} q^{C 2^{n_{1}}\left|x-t_{0}-2^{-j-1}\right|} .
$$

Supposing again that $x-t_{0} \in\left[2^{-k}, 2^{-k+1}\right.$ ) for some $1 \leq k \leq K$, we get

$$
\begin{aligned}
C|I|^{2} 2^{n_{1}} \sum_{i=0}^{n_{1}-1} \sum_{j=k}^{i} 2^{j} q^{C 2^{n_{1}}\left|x-t_{0}-2^{-j-1}\right|} & \leq C|I|^{2} \sum_{i=0}^{n_{1}-1} 2^{i-n_{1}} 2^{2 n_{1}} q^{C 2^{n_{1}}\left|x-t_{0}\right|} \\
& \leq C|I|^{2} \sum_{i=0}^{n_{1}-1} 2^{i-n_{1}}\left|x-t_{0}\right|^{-2} \\
& \leq C|I|^{2}\left|x-t_{0}\right|^{-2} .
\end{aligned}
$$

To investigate the remaining term, observe that

$$
\begin{aligned}
& C|I|^{2} 2^{n_{1}} \sum_{i=0}^{\left(n_{1}-1\right) \wedge(k-1)} \sum_{j=0}^{(k-1) \wedge i} 2^{j} q^{C 2^{n_{1}}\left|x-t_{0}-2^{-j-1}\right|} \\
& \leq C|I|^{2} \sum_{j=0}^{\left(n_{1}-1\right) \wedge(k-1)} \sum_{i=j}^{\left(n_{1}-1\right) \wedge(k-1)} 2^{\left(j-n_{1}\right) \varepsilon} 2^{j(1-\varepsilon)} 2^{n_{1}(1+\varepsilon)} q^{C 2^{n_{1}}\left|x-t_{0}-2^{-j-1}\right|} \\
& \leq C|I|^{2} \sum_{j=0}^{k-1} 2^{j(1-\varepsilon)}\left|x-t_{0}-2^{-j-1}\right|^{-(1+\varepsilon)},
\end{aligned}
$$

where $0<\varepsilon<1$ is to be chosen later. Moreover, if $k<n$ then

$$
\begin{aligned}
C|I|^{2} 2^{n_{1}} \sum_{i=k}^{n_{1}-1} \sum_{j=0}^{(k-1) \wedge i}{ }^{j} q^{C 2^{n_{1}}}\left|x-t_{0}-2^{-j-1}\right| \\
\leq C|I|^{2} \sum_{j=0}^{k-1} \sum_{i=k}^{n_{1}-1} 2^{\left(j-n_{1}\right) \varepsilon} 2^{j(1-\varepsilon)}\left|x-t_{0}-2^{-j-1}\right|^{-(1+\varepsilon)} \\
\leq C|I|^{2} \sum_{j=0}^{k-1} 2^{j(1-\varepsilon)}\left|x-t_{0}-2^{-j-1}\right|^{-(1+\varepsilon)} .
\end{aligned}
$$

Since $p<1$ we can choose $\varepsilon$ such that $(1+\varepsilon) p<1$. Consequently,

$$
\begin{gathered}
\int_{(16 I)^{\mathrm{c}}} \sup _{n \geq 2^{K}}\left(\int_{I} D_{2, n}(t, x) d t\right)^{p} d x \leq C_{p}|I|^{2 p} \int_{(16 I)^{\mathrm{c}}}\left|x-t_{0}\right|^{-2 p} d x \\
+C_{p}|I|^{2 p} \sum_{k=1}^{K} \sum_{j=0}^{k-1} 2^{j(1-\varepsilon) p}\left|x-t_{0}-2^{-j-1}\right|^{-(1+\varepsilon) p} d x \\
\leq C_{p}|I|+C_{p}|I|^{2 p} \sum_{k=1}^{K} \sum_{j=0}^{k-1} 2^{j(1-\varepsilon) p} 2^{-j(1-(1+\varepsilon) p)} \leq C_{p}|I| .
\end{gathered}
$$


Obviously,

$$
E_{n}(t, x)=E_{1, n}(t, x)+E_{2, n}(t, x)
$$

where

$$
\begin{aligned}
E_{1, n}(t, x):= & C 2^{-n_{1}} \sum_{i=K}^{n_{1}-1} 2^{i} \sum_{j=0}^{i} 2^{j} \\
& \times \sum_{l=-2^{n_{1}-i}+1}^{2^{n_{1}-i}-1} 1_{\left\{2^{-j-1}+8 I\right\}}(x) q^{2^{n_{1}}\left|x-t-2^{-j-1}-l / 2^{n_{1}}\right|} \\
E_{2, n}(t, x):= & C 2^{-n_{1}} \sum_{i=K}^{n_{1}-1} 2^{i} \sum_{j=0}^{i} 2^{j} \\
& \times \sum_{l=-2^{n_{1}-i}+1}^{2^{n_{1}-i}-1} 1_{\left\{2^{-j-1}+8 I\right\}}(x) q^{2^{n_{1}}\left|x-t-2^{-j-1}-l / 2^{n_{1}}\right|} .
\end{aligned}
$$

We obtain

$$
E_{1, n}(t, x) \leq C \sum_{j=0}^{K-1} 2^{j} 1_{\left\{2^{-j-1}+8 I\right\}}(x)
$$

and so

$$
\int_{(16 I)^{\mathrm{c}}} \sup _{n \geq 2^{K}}\left(\int_{I} E_{1, n}(t, x) d t\right)^{p} d x \leq C_{p}|I| .
$$

Finally,

$$
E_{2, n}(t, x) \leq C 2^{-K} \sum_{i=K}^{n_{1}-1} 2^{i} \sum_{j=0}^{i} 2^{j} 2^{n_{1}-i} q^{C 2^{n_{1}}\left|x-t_{0}-2^{-j-1}\right|}
$$

and

$$
\int_{(16 I)^{\mathrm{c}}} \sup _{n \geq 2^{K}}\left(\int_{I} E_{2, n}(t, x) d t\right)^{p} d x \leq C_{p}|I|
$$

can be shown as (26). This finishes the proof of (14).

For (16) suppose that $n<2^{K}$ and $N=1$. We estimate the last term of (24) by

$$
F_{n}(t, x):=C \sum_{i=0}^{n_{1}-1} 2^{i} \sum_{j=0}^{i} 2^{j} \sum_{l=-2^{n_{1}-i}+1}^{2^{n_{1}-i}-1} q^{2^{n_{1}}\left|x-t-2^{-j-1}-l / 2^{n_{1}}\right|} .
$$

Comparing this expression with the definition of $D_{n}(t, x)$ we obtain the inequality

$$
\int_{(16 I)^{\mathrm{c}}} \sup _{n<2^{K}}\left(\int_{I} F_{n}(t, x) d t\right)^{p} d x \leq C_{p}|I|^{1-p},
$$

which verifies (16). The proof of the lemma is complete. 
Lemma 2. If $m \geq 0$ and $|k| \leq m+1$ then

$$
\begin{array}{r}
\int_{0}^{1} \frac{1}{n} \sum_{i=0}^{n_{1}-1}\left|L_{i}^{(m, k)}(t, x)\right| d t \leq C, \\
\quad \int_{0}^{1} \frac{1}{n}\left|M_{n}^{(m, k)}(t, x)\right| d t \leq C .
\end{array}
$$

Proof. Writing $N=0$ in (18) and integrating in $t$ we conclude that

$$
\int_{0}^{1} \frac{1}{n} \sum_{i=0}^{n_{1}-1}\left|L_{i}^{(m, k)}(t, x)\right| d t \leq C 2^{-n_{1}} \sum_{i=0}^{n_{1}-1} 2^{i} \sum_{j=0}^{i} 2^{j} 2^{-i} \leq C .
$$

Inequality (28) can be proved similarly from (24).

Now we are ready to prove our main theorem.

Theorem 2. If $m \geq 0$ and $|k| \leq m+1$ then

$$
\left\|\sigma_{*}^{(m, k)} f\right\|_{p} \leq C_{p}\|f\|_{H_{p}} \quad\left(f \in H_{p}\right)
$$

for all $1 / 2<p<\infty$. In particular, if $f \in L_{1}$ then

$$
\lambda\left(\sigma_{*}^{(m, k)} f>\varrho\right) \leq \frac{C}{\varrho}\|f\|_{1} \quad(\varrho>0) .
$$

Proof. Theorem 1 implies

$$
\begin{aligned}
\left|\sigma_{n}^{(m, k)} f(x)\right| \leq & \frac{2}{n} \sum_{i=0}^{n_{1}} 2^{i}\left|C_{2^{i}}^{(m, k)} f(x)\right|+\frac{1}{n} \sum_{i=0}^{n_{1}-1}\left|\int_{0}^{1} L_{i}^{(m, k)}(t, x) f(t) d t\right| \\
& +\frac{1}{n}\left|\int_{0}^{1} M_{n}^{(m, k)}(t, x) f(t) d t\right| .
\end{aligned}
$$

We denote the second and third term on the right hand side by $A_{n}^{(m, k)} f(x)$ and $B_{n}^{(m, k)} f(x)$, respectively. Since $C_{2^{n}}^{(m, k)} f=P_{2^{n}}^{(m, k)} f$, we have

$$
\left|\sigma_{*}^{(m, k)} f\right| \leq 4 P_{*}^{(m, k)} f+\sup _{n \in \mathbb{N}} A_{n}^{(m, k)} f+\sup _{n \in \mathbb{N}} B_{n}^{(m, k)} f .
$$

By Theorem A and (11) the proof of the theorem will be complete if we show that the operators $\sup _{n \in \mathbb{N}} A_{n}^{(m, k)}$ and $\sup _{n \in \mathbb{N}} B_{n}^{(m, k)}$ are bounded on $L_{\infty}$ and are $p$-quasi-local for each $1 / 2<p<1$.

The boundedness follows from Lemma 2 . Choose a $p$-atom $a$ with support $I$ and assume that $2^{-K-1}<|I| \leq 2^{-K}(K \in \mathbb{N})$. It follows from the definition of the atom and from (13) that 


$$
\begin{aligned}
& \int_{(16 I)^{\mathrm{c}}} \sup _{n \geq 2^{K}}\left|A_{n}^{(m, k)} a(x)\right|^{p} d x \\
& \quad \leq|I|^{-1} \int_{(16 I)^{\mathrm{c}}} \sup _{n \geq 2^{K}}\left(\int_{I} \frac{1}{n} \sum_{i=0}^{n_{1}-1}\left|L_{i}^{(m, k)}(t, x)\right| d t\right)^{p} d x \leq C_{p} .
\end{aligned}
$$

Now let $n<2^{K}$. If $k=m+1$ then it is easy to see that $L_{i}^{(m, k)}(t, x)$ $\left(i=0, \ldots, n_{1}-1\right)$ and $M_{n}^{(m, k)}(t, x)$ is constant on the dyadic interval $I$ and so $A_{n}^{(m, k)} a=0$ and $B_{n}^{(m, k)} a=0\left(n<2^{K}\right)$. Therefore we can suppose that $k \leq m$. For

$$
A(x):=\int_{0}^{x} a(t) d t
$$

we have $\operatorname{supp} A \subset I, A$ is zero at the endpoints of $I$ and $\|A\|_{\infty} \leq|I|^{1-1 / p}$. Integrating by parts we can see that

$$
A_{n}^{(m, k)} a(x)=\frac{1}{n} \sum_{i=0}^{n_{1}-1}\left|\int_{I} D_{t} L_{i}^{(m, k)}(t, x) A(t) d t\right| .
$$

Thus (15) implies

$$
\begin{aligned}
& \int_{(16 I)^{\mathrm{c}}} \sup _{n<2^{K}}\left|A_{n}^{(m, k)} a(x)\right|^{p} d x \\
& \quad \leq|I|^{p-1} \int_{(16 I)^{\mathrm{c}}} \sup _{n<2^{K}}\left(\int_{I} \frac{1}{n} \sum_{i=0}^{n_{1}-1}\left|D_{t} L_{i}^{(m, k)}(t, x)\right| d t\right)^{p} d x \leq C_{p},
\end{aligned}
$$

which proves the $p$-quasi-locality of $\sup _{n \in \mathbb{N}} A_{n}^{(m, k)}$. Notice that by interpolation we can suppose that $p<1$. With the help of Lemma 1 the $p$-quasilocality of $\sup _{n \in \mathbb{N}} B_{n}^{(m, k)}$ can be shown in the same way.

We note that (30) for the Walsh system is due to Schipp [9] (see also Weisz [16]).

Observe that since $P_{*}^{(m, k)}$ is bounded on $L_{\infty}$ (see Weisz [14]), we have

$$
\left\|\sigma_{*}^{(m, k)} f\right\|_{\infty} \leq C\|f\|_{\infty} \quad\left(f \in L_{\infty}\right) .
$$

The usual density argument gives

Corollary 1. If $m \geq 0$ and $|k| \leq m+1$ then $f \in L_{1}$ implies

$$
\sigma_{n}^{(m, k)} f \rightarrow f \quad \text { a.e. as } n \rightarrow \infty .
$$

This convergence result for the Walsh system is due to Fine [7] (see also Schipp [9] and Weisz [16]). 


\section{References}

[1] S. V. Bochkarev, A Fourier series that diverges on a set of positive measure for an arbitrary bounded orthonormal set, Mat. Sb. 98 (1975), 436-449 (in Russian).

[2] Z. Ciesielski, A bounded orthonormal system of polygonals, Studia Math. 31 (1968), 339-346.

[3] - Constructive function theory and spline systems, ibid. 53 (1975), 277-302.

[4] —, Equivalence, unconditionality and convergence a.e. of the spline bases in $L_{p}$ spaces, in: Approximation Theory, Banach Center Publ. 4, PWN-Polish Sci. Publ., Warszawa, 1979, 55-68.

[5] Z. Ciesielski and J. Domsta, Construction of an orthonormal basis in $C^{m}\left(I^{d}\right)$ and $W_{p}^{m}\left(I^{d}\right)$, Studia Math. 41 (1972), 211-224.

[6] Z. Ciesielski, P. Simon, and P. Sjölin, Equivalence of Haar and Franklin bases in $L_{p}$ spaces, ibid. 60 (1977), 195-210.

[7] N. J. Fine, Cesàro summability of Walsh-Fourier series, Proc. Nat. Acad. Sci. U.S.A. 41 (1955), 558-591.

[8] K. S. Kazarian and A. S. Sargsian, Divergence a.e. of the Fourier series with respect to the Ciesielski system, Izv. Akad. Nauk Armyan. SSR 24 (1989), 403-412 (in Russian).

[9] F. Schipp, Über gewissen Maximaloperatoren, Ann. Univ. Sci. Budapest Sect. Math. 18 (1975), 189-195.

[10] -, On a.e. convergence of expansion with respect to a bounded orthonormal system of polygonals, Studia Math. 58 (1976), 287-290.

[11] F. Schipp, W. R. Wade, P. Simon, and J. Pál, Walsh Series: An Introduction to Dyadic Harmonic Analysis, Adam Hilger, Bristol, 1990.

[12] P. Sjölin and J.-O. Strömberg, Basis properties of Hardy spaces, Ark. Mat. 21 (1983), 111-125.

[13] E. M. Stein, Harmonic Analysis: Real-Variable Methods, Orthogonality, and Oscillatory Integrals, Princeton Univ. Press, Princeton, NJ, 1993.

[14] F. Weisz, Multi-parameter unbounded Ciesielski systems and convergence results, East J. Approx. 6 (2000), 397-420.

[15] - Martingale Hardy Spaces and their Applications in Fourier-Analysis, Lecture Notes in Math. 1568, Springer, Berlin, 1994.

[16] - Cesàro summability of one- and two-dimensional Walsh-Fourier series, Anal. Math. 22 (1996), 229-242.

[17] P. Wojtaszczyk, $H_{p}$-spaces, $p \leq 1$, and spline systems, Studia Math. 77 (1984), 289-320.

Department of Numerical Analysis

Eötvös L. University

Pázmány P. sétány 1/D

H-1117 Budapest, Hungary

E-mail: weisz@ludens.elte.hu

Received June 11, 1999

Revised version January 7, 2000 